\title{
Anticancer secondary metabolites from marine sponges
}

\section{Deniz süngerlerinden antikanser metabolitler}

\author{
İbrahim Seyda Uras ${ }^{1}$ - Belma Konuklugil ${ }^{i^{*}}$ \\ 1 Department of Pharmacognosy, Faculty of Pharmacy, Ağrı Ibrahim Çeçen University, Ağrı, Turkey \\ 2 Department of Pharmacognosy, Faculty of Pharmacy, Ankara University, Ankara, Turkey \\ https://orcid.org/0000-0001-5945-8743 \\ iD https://orcid.org/0000-0002-4753-0450
}

\author{
How to cite this paper: \\ Uras, I.S. \& Konuklugil, B. (2021). Anticancer secondary metabolites from marine sponges. Ege Journal of Fisheries and Aquatic Sciences, 38(1), $101-106$. \\ DOI: 10.12714/egejfas.38.1.12
}

Abstract: The oceans cover $70 \%$ of the Earth's surface. The marine environment is an important source of secondary metabolites with high biodiversity. Besides other marine species, sponges with a wide range of secondary metabolites are an important potential for drug discovery. Cancer is one of the leading causes of death with high morbidity and mortality. It is very important to discover new therapeutic agents in the treatment of cancer. In recent years, studies on exploring new anticancer compounds are focused on the marine source. In this review, our target is collecting the studies about marine sponges secondary metabolites which have an anticancer effect. Among most of the isolated compounds from sponges and their semisynthetic derivatives, there are three FDA (US Food and Drug Administration) approved compounds and three compounds in clinical phase. Moreover, more than 40 compounds isolated from marine sponges have been tested for anticancer activity in recent 10 years. In conclusion marine sponges secondary metabolites are a promising and important source of the anticancer compounds.

Keywords: Natural products, marine sponges, secondary metabolites, cancer treatment

Öz: Okyanuslar dünya yüzeyinin 70\%'ini kaplamaktadır. Deniz ortamı, yüksek biyolojik çeşitlilik sunan, önemli bir sekonder metabolit kaynağıdır. Diğer deniz türlerinin yanı sıra, çok çeşitli sekonder metabolitlere sahip olan süngerler, ilaç keşfi için önemli bir potansiyel taşımaktadır. Kanser, yüksek morbidite ve mortalite ile önde gelen ölüm nedenlerinden biridir. Kanser tedavisinde yeni terapötik ajanların bulunması çok önemlidir. Son yıllarda, yeni antikanser bileşiklerini keşfetmeye yönelik çalışmalar deniz kaynaklarına odaklanmıştır. Bu derlemede hedefimiz antikanser etkisi olan, deniz süngerinden izole edilen sekonder metabolitler ile ilgili çalışmaları toplamaktır. Süngerlerden elde edilen bileşikler ve bu bileşiklerin yarısentetik türevleri arasından 3 adet bileşik FDA (Amerika Gıda ve İlaç Dairesi) tarafından onaylanmış ve 3 bileşik klinik faz çalışmalarındadır. Ayrıca, deniz süngerlerinden izole edilmiş 40'tan fazla bileşik, son 10 yılda antikanser aktivitesi açısından test edilmiştir. Sonuç olarak deniz süngerlerinden elde edilen sekonder metabolitler önemli ve gelecek vaat eden bir antikanser bileşik kaynağıdır.

Anahtar kelimeler: Doğal ürünler, deniz süngerleri, sekonder metabolitler, kanser tedavisi

\section{INTRODUCTION}

Cancer is a major global health problem responsible for 13 million deaths worldwide, and death rate is expected to rise to 13.1 million by 2030 . Despite the progress in the field of cancer research, there is still a need to discover and develop anticancer therapeutic agents (Torre et al., 2015).

Natural products from plants, animals, and marine sources have been used for the treatment of different diseases and are becoming an important research area for drug discovery. Generally, they are used by the producing organisms to compete for vital resources, defend against predators and communicate interspecies (Dias et al., 2012; Mann, 1994). These products have been extensively studied and have shown some bio-activities such as antimicrobial, anti-inflammatory, antiprotozoal activity and anticancer activity. The natural source based drug discovery resulted mainly in the development of anticancer agents from plants (vincristine, vinblastine, etoposide, paclitaxel, camptothecin, topotecan and irinotecan), marine organisms (citarabine, aplidine and dolastatin) and micro-organisms (dactinomycin, bleomycin and doxorubicin (Thomas et al., 2010; L. Wang et al., 2012).

In the last decades, marine organisms are accepted as a new source of novel molecules for new drug discovery and drug development. The large diversity of the marine habitats led the marine invertebrates to produce wide range of bioactive compounds such as acids, alkaloids, esters, fatty acids, glycosides, lipids, peptides, terpenes, terpenoids, with therapeutic effect (Malve, 2016). Besides, the marine ecological environment has unique characteristics like, low level oxygen and light, high salt, extreme temperatures and high pressure. Due to these difficulties, enzyme reaction system and metabolic pathways of marine organisms are remarkably different from terrestrial counterparts (Schumacher et al., 2011).

Marine natural products provide a rich source of chemically diverse compounds which have significant potential to develop novel types of anticancer agents. The biodiversity of marine life and the accompanying chemical diversity enable the marine environment to possess infinite 
bioactive secondary metabolites (Pihlanto-Leppälä, 2000). Bioactive secondary metabolites are isolated from such sources as corals, sponges, marine plants, animals and microorganisms (Rasmussen and Morrissey, 2007). These isolated bioactive secondary metabolites have been found to be effective as antifungal, neuroprotective, anti-tumor, photoprotective, antibiotic, and anti-infective bioactivities (Bérdy, 2005; Mishra and Tiwari, 2011; Molinski et al., 2009; Pettit et al., 1982; Sudek et al., 2007).

Owing to their various and significant bioactivities, marine natural products have become important for pharmaceutical and cosmetic industries. There are eight drugs from marine source on the market with FDA or EMEA (European Medicines Agency) approval (Figure 1) (Martins et al., 2014). Among these drugs, Halaven was obtained from sponges.

Number of new terpenoids from invertebrates is increasing, contrasting with the decreasing trend in the discovery of new alkaloids and aliphatic molecules (Leal et al., 2012). These secondary metabolites are important in the development of compounds to treat cancer. There are three compounds already approved by FDA for cancer treatment isolated from sponges (Table 1) and two secondary metabolites are in clinical trials at various stages (Table 2).

Besides the other marine organisms, sponges have ability to produce important quantity of secondary metabolites possessing unique structures with certain activities. Due to

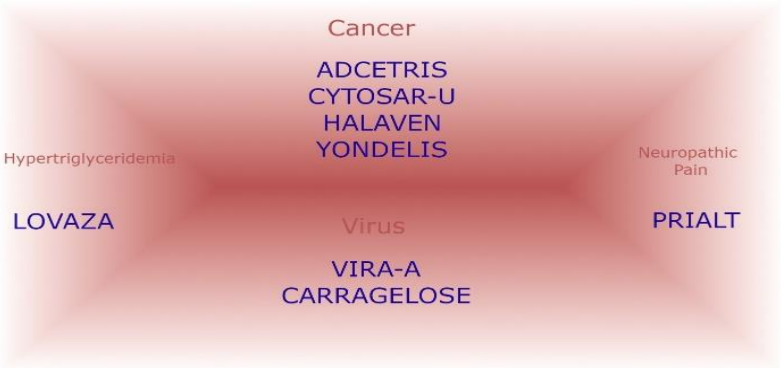

Figure 1. Eight drugs with FDA or EMEA approval from marine source on the market for different diseases (Martins et al., 2014)

the lack of a protective shell, immune system and mobility, sponges have developed an ability to synthesize different compounds to survive. For this reason, sponges are considered as the focus of natural product studies for a long time (Livett et al., 2004). Up to date approximately, 8000 sponge species were identified, and almost two times not identified. Due to the fact that many natural resources have been investigated, attention moved to marine environment and specifically to marine microorganisms like cyanobacteria, sponge, fungi and several other groups of marine bacteria because of their wide diversity (Bhatnagar and Kim, 2010).

Table 1. FDA Approved anticancer aompounds isolated from sponges

\begin{tabular}{lllll}
\hline Compound & Source of Metabolite & Chemical Class & Clinical Status & Reference \\
\hline Eribulin Mesylate (Halaven) & Lissodendoryx sp. & Macrolide & FDA Approved & (Menis and Twelves, 2011) \\
Cytarabine (Ara-C) & Cryptotheca crypta & Nucleoside & FDA Approved & (Krauss et al., 2018) \\
Gemcitabine & Tectitethya crypta & Nucleoside & FDA Approved & (Gesto et al., 2012) \\
\hline
\end{tabular}

Table 2. Currently in Clinical Pipeline Anticancer Compounds Isolated from Sponges

\begin{tabular}{lllll}
\hline Compound & Source of Metabolite & Chemical Class & Clinical Status & Reference \\
\hline Plocabulin (PM060184) & Lithoplocamia lithistoides & Polyketide & Phase II & (Pantazopoulou et al., 2018) \\
MORAb-202 (antibody-drug conjugate) & Halichondria okadai & Macrolide & Phase I & (Cheng et al., 2018) \\
\hline
\end{tabular}

\section{RESULTS AND DISCUSSION}

Marine sponges are still a virgin area. Studies should be concentrated in this area, especially since living organisms in the marine environment are important for the discovery of new metabolites. In the light of the given data it can be seen that many secondary metabolites are obtained from marine sponges. When these compounds are tested for cancer treatment, some of the compounds showed significant activity. Potential of these compounds are evaluating by clinical stages to product new drugs (Table 3).

It is hoped that the secondary metabolites, which have shown considerable success in preclinical experiments, can be used in the treatment of cancer by feeding the clinical experiment process. 
Table 3. List of secondary metabolites which tested for anti cancer activity in last 10 years

\begin{tabular}{|c|c|c|c|c|}
\hline Secondary Metabolite & Source of Metabolite & Cell Line & Concentration Range & Reference \\
\hline Isoaaptamine & Aaptos sp. & THP-1 & $10-25 \mu \mathrm{M}$ & (Dyshlovoy et al., 2014) \\
\hline Aaptamine & Aaptos aaptos & THP-1 & $50-200 \mu \mathrm{M}$ & (Dyshlovoy et al., 2014) \\
\hline Microsclerodermin A & Amphibleptula sp. & $\begin{array}{l}\text { AsPC-1 } \\
\text { BxPC-3 }\end{array}$ & $\begin{array}{l}2.4 \mu \mathrm{M} \\
2.4 \mu \mathrm{M}\end{array}$ & (Guzman et al., 2015) \\
\hline Isofistularin-3 & Aplysina aerophoba & U937 & $50 \mu \mathrm{M}$ & (Florean et al., 2016) \\
\hline Crambescidin 800 & Crambe crambe & $\begin{array}{l}\text { Raji } \\
\text { HepG2 }\end{array}$ & $\begin{array}{l}50 \mu \mathrm{M} \\
0.5-2.5 \mu \mathrm{M}\end{array}$ & (Roel etal 2016) \\
\hline Salarin C. & Fascaplysinopsis sp. & K562 & $0.01-0.2 \mu \mathrm{M}$ & (Ben-Califa et al., 2012) \\
\hline Fascaplysin & Fascaplysinopsis Bergquist sp. & $\begin{array}{l}\text { A549 } \\
\text { OVCAR3 } \\
\text { S457 }\end{array}$ & $\begin{array}{l}0.63 \mu \mathrm{M} \\
0.52 \mu \mathrm{M} \\
0.2 \mu \mathrm{M}\end{array}$ & (Rath et al., 2018) \\
\hline \multirow{5}{*}{$\begin{array}{l}\text { llimaquinone } \\
\text { Heteronemin }\end{array}$} & \multirow{5}{*}{$\begin{array}{l}\text { Hippospongia metachromia } \\
\text { Hyrtios sp. }\end{array}$} & \multirow{5}{*}{$\begin{array}{l}\text { HCT116 } \\
\text { K562 } \\
\text { DU145 } \\
\text { PC-3 } \\
\text { LNCaP } \\
\text { T24 } \\
\text { A498 }\end{array}$} & \multirow{5}{*}{$\begin{array}{l}2.5-10 \mu \mathrm{M} \\
1.4-5.6 \mu \mathrm{M} \\
0.01-1 \mu \mathrm{gL} / \mathrm{mL} \\
0.01-1 \mu \mathrm{g} / \mathrm{mL} \\
0.01 \mu \mathrm{g} / \mathrm{mL} \\
0.1-0.8 \mu \mathrm{g} / \mathrm{mL} \\
0.5-3 \mu \mathrm{M}\end{array}$} & (Lee et al., 2015) \\
\hline & & & & (Schumacher et al., 2010) \\
\hline & & & & (Wu et al., 2016) \\
\hline & & & & (Huang et al., 2016) \\
\hline & & & & \\
\hline Stellettin B & Jaspis stellifera & $\begin{array}{l}\text { K562 } \\
\text { A549 } \\
\text { SF295 }\end{array}$ & $\begin{array}{l}0.012-0.054 \mu \mathrm{M} \\
0.02-1 \mu \mathrm{M} \\
0.04-1 \mu \mathrm{M}\end{array}$ & $\begin{array}{l}\text { (Chen et al., 2017) } \\
\text { (Wang et al., 2016) } \\
\text { (Tang et al., 2014) }\end{array}$ \\
\hline \multirow{4}{*}{$\begin{array}{l}\text { Leiodermatolide } \\
\text { Monanchocidin A } \\
\text { Monanchocidin B } \\
\text { Rhizocalinin }\end{array}$} & \multirow{4}{*}{$\begin{array}{l}\text { Leiodermatium sp. } \\
\text { Monanchora pulchra } \\
\text { Monanchora pulchra } \\
\text { Rhizochalina incrustata }\end{array}$} & AsPC-1 & $0.01 \mu \mathrm{M}$ & \multirow{3}{*}{$\begin{array}{l}\text { (Guzman et al., 2016) } \\
\text { (Dyshlovoy et al., 2016a) }\end{array}$} \\
\hline & & HeLa & $1.39 \mu \mathrm{M}$ & \\
\hline & & HeLa & $0.58 \mu \mathrm{M}$ & \\
\hline & & $\begin{array}{l}\text { HL-60 } \\
\text { HT-29 } \\
\text { THP-1 } \\
\text { PC-3 } \\
\text { DU-145 } \\
\text { 22Rv1 } \\
\text { VCaP }\end{array}$ & $\begin{array}{l}10-25 \mu \mathrm{M} \\
1-6 \mu \mathrm{M} \\
1-10 \mu \mathrm{M} \\
0.5-4 \mu \mathrm{M} \\
0.5-4 \mu \mathrm{M} \\
0.5-4 \mu \mathrm{M} \\
0.5-4 \mu \mathrm{M}\end{array}$ & $\begin{array}{l}\text { (Jin et al., 2009) } \\
\text { (Khanal et al., 2011) } \\
\text { (Fedorov et al., 2009) } \\
\text { (Dyshlovoy et al., 2016b) }\end{array}$ \\
\hline Sipholenol A & Siphonochalina sp. & $\begin{array}{l}\text { HepG2 } \\
\text { HCT-116 }\end{array}$ & $\begin{array}{l}17.18 \mu \mathrm{M} \\
14.8 \mu \mathrm{M}\end{array}$ & \multirow{2}{*}{$\begin{array}{l}\text { (Abdel-Lateff et al., 2016) } \\
\text { (Sobahi et al., 2017) }\end{array}$} \\
\hline Sipholenol L & Siphonochalina sp. & $\begin{array}{l}\text { HepG2 } \\
\text { HCT-116 }\end{array}$ & $\begin{array}{l}24 \mu \mathrm{M} \\
19.8 \mu \mathrm{M}\end{array}$ & \\
\hline Spongiatriol & Australian spongia sp. & $\begin{array}{l}\text { AsPC-1 } \\
\text { PANC-1 } \\
\text { MIA PaCa-2 } \\
\text { BxPC-3 }\end{array}$ & $\begin{array}{l}6.8 \mu \mathrm{M} \\
6.8 \mu \mathrm{M} \\
6.8 \mu \mathrm{M} \\
6.8 \mu \mathrm{M}\end{array}$ & (Guzman et al., 2013) \\
\hline Scalaradial & Cacospongia scalaris & $\begin{array}{l}\text { HeLa } \\
\text { T47D }\end{array}$ & $\begin{array}{l}10 \mu \mathrm{g} / \mathrm{mL} \\
10 \mu \mathrm{g} / \mathrm{mL}\end{array}$ & (De Stefano et al., 2012) \\
\hline \multirow[t]{2}{*}{$\begin{array}{l}\text { Callyspongidiol } \\
\text { Lasonolide A }\end{array}$} & $\begin{array}{l}\text { Callyspongia sp. } \\
\text { Forcepia sp. }\end{array}$ & $\begin{array}{l}\mathrm{HL}-60 \\
\text { CA46 }\end{array}$ & $\begin{array}{l}31.0-77.5 \mu \mathrm{M} \\
0.1 \mu \mathrm{M}\end{array}$ & \multirow[t]{2}{*}{$\begin{array}{l}\text { (Umeyama et al., 2010) } \\
\text { (Zhang et al., 2012) }\end{array}$} \\
\hline & & $\begin{array}{l}\text { MCF-7 } \\
\text { HCT-116 }\end{array}$ & $\begin{array}{l}0.1 \mu \mathrm{M} \\
0.1 \mu \mathrm{M}\end{array}$ & \\
\hline Geoditin A & Geodia japonica & $\begin{array}{l}\text { HL-60 } \\
\text { HT-29 }\end{array}$ & $\begin{array}{l}1.6 \text { to } 25 \mu \mathrm{g} / \mathrm{mL} \\
5-30 \mu \mathrm{M}\end{array}$ & \multirow{3}{*}{$\begin{array}{l}\text { (Liu et al., 2005) } \\
\text { (Cheung et al., 2010) } \\
\text { (Schneiders et al., 2009) } \\
\text { (Rothmeier et al., 2010) } \\
\text { (Chinen et al., 2010) }\end{array}$} \\
\hline Spongistatin 1 & Hyrtios erecta & $\begin{array}{l}\text { MCF-7 } \\
\text { L3.6pl }\end{array}$ & $\begin{array}{l}0.0002-0.0005 \mu \mathrm{M} \\
0.00001-0.01 \mu \mathrm{M}\end{array}$ & \\
\hline Irciniastatin A & $\begin{array}{l}\text { Ircinia ramose } \\
\text { Psammocinia sp. }\end{array}$ & Jurkat & $0.01 \mu \mathrm{M}$ & \\
\hline Jaspine B & Jaspis sp. & $\begin{array}{l}\mathrm{B} 16 \\
\mathrm{HaCaT}\end{array}$ & $\begin{array}{l}5 \mu \mathrm{M} \\
5 \mu \mathrm{g} / \mathrm{mL}\end{array}$ & \multirow{2}{*}{$\begin{array}{l}\text { (Salma et al., 2009) } \\
\text { (Yoo et al., 2012) } \\
\text { (Nguyen et al., 2009) }\end{array}$} \\
\hline $\begin{array}{l}\text { Petrosterol-3,6-dione } \\
\text { 5,6-epoxy-petrosterol }\end{array}$ & $\begin{array}{l}\text { Lanthella sp. } \\
\text { Lanthella sp. }\end{array}$ & $\begin{array}{l}\mathrm{HL}-60 \\
\mathrm{HL}-60\end{array}$ & $\begin{array}{l}19.9 \mu \mathrm{M} \\
21.3 \mu \mathrm{M}\end{array}$ & \\
\hline $\begin{array}{l}\text { Petrosterol } \\
\text { Latrunculin A }\end{array}$ & $\begin{array}{l}\text { Lanthella sp. } \\
\text { Negombata magnifica }\end{array}$ & $\begin{array}{l}\text { HL-60 } \\
\text { NUGC-4 }\end{array}$ & $\begin{array}{l}21.5 \mu \mathrm{M} \\
0.01-10 \mu \mathrm{M}\end{array}$ & (Konishi et al., 2009) \\
\hline Psammaplysene A & Psammaplysilla sp. & $\begin{array}{l}\text { ECC1 } \\
\text { Ishikawa }\end{array}$ & & (Berry et al., 2009) \\
\hline $\begin{array}{l}\text { Smenamides A and B } \\
\text { Renieramycin M } \\
\text { Isobatzelline E } \\
\text { Calyculin A }\end{array}$ & $\begin{array}{l}\text { Smenospongia aurea } \\
\text { Xestospongia sp. } \\
\text { Batzella sp. } \\
\text { Discodermia calyx }\end{array}$ & $\begin{array}{l}\text { Calu-1 } \\
\text { H460 } \\
\text { AsPC-1 } \\
\text { MDA-MB-468 } \\
\text { MCF-7 } \\
\text { MDA-MB-231 }\end{array}$ & $\begin{array}{l}0.05-0.1 \mu \mathrm{M} \\
5-40 \mu \mathrm{M} \\
5 \text { or } 25 \mu \mathrm{g} / \mathrm{mL} \\
0.01 \mu \mathrm{M} \\
0.01 \mu \mathrm{M} \\
0.01 \mu \mathrm{M}\end{array}$ & $\begin{array}{l}\text { (Teta et al., 2013) } \\
\text { (Halim et al., 2011) } \\
\text { (Guzman et al., 2009) } \\
\text { (Edelson and Brautigan, 2011) }\end{array}$ \\
\hline $\begin{array}{l}\text { (19Z)-Halichondramide } \\
\text { Leiodermatolide }\end{array}$ & $\begin{array}{l}\text { Chondrosia corticata } \\
\text { Leiodermatium sp. }\end{array}$ & $\begin{array}{l}\text { A549 } \\
\text { A549 } \\
\text { U20S }\end{array}$ & $\begin{array}{l}0.025-0.1 \mu \mathrm{M} \\
0.01-1 \mu \mathrm{M} \\
0.018-0.23 \mu \mathrm{M}\end{array}$ & \multirow{5}{*}{$\begin{array}{l}\text { (Bae et al., 2013) } \\
\text { (Paterson et al., 2011) } \\
\text { (Mailhol et al., 2014) } \\
\text { (Chan et al., 2011) } \\
\text { (W. Wang et al., 2009) } \\
\text { (Radwan et al., 2012) } \\
\text { (Su et al., 2012) } \\
\text { (Kong et al., 2008) } \\
\text { (Whitson et al., 2011) } \\
\text { (Ebada et al., 2009) }\end{array}$} \\
\hline \multirow{4}{*}{$\begin{array}{l}\text { Peloruside A } \\
\text { FBA-TPQ } \\
\text { Manzamine A } \\
\text { 10-Acetylirciformonin B } \\
\text { Smenospongine } \\
\text { Candidaspongiolide A/B } \\
\text { Jaspamide }\end{array}$} & $\begin{array}{l}\text { Mycale hentscheli } \\
\text { Zyzzya sp. } \\
\text { Haliclona sp. }\end{array}$ & $\begin{array}{l}\text { MCF-7 } \\
\text { MCF-7 } \\
\text { AsPC-1 }\end{array}$ & $\begin{array}{l}0.025-0.1 \mu \mathrm{MM} \\
2.297 \mathrm{mumo} / \mathrm{L}\end{array}$ & \\
\hline & $\begin{array}{l}\text { Ircinia sp. } \\
\text { Snonia }\end{array}$ & $\begin{array}{l}\text { HL-60 } \\
\text { MCF-7-Nanng }\end{array}$ & $2.5 \mathrm{~g} / \mathrm{mL}$ & \\
\hline & $\begin{array}{l}\text { Spongia pertusa Esper } \\
\text { Candidaspongia sp. }\end{array}$ & $\begin{array}{l}\text { MCF-7-Nanog } \\
\text { MCF-7 }\end{array}$ & $\begin{array}{l}6.06 \mu \mathrm{MM} \\
2.0 \mathrm{nM}\end{array}$ & \\
\hline & Jaspis johnstoni & L5178Y & $0.1 \mu \mathrm{g} / \mathrm{mL}$ & \\
\hline
\end{tabular}




\section{CONCLUSION}

Sponges are one of the most interesting taxa of marine organisms with respect to the discovery of active compounds for the pharmaceutical application. In conclusion, it has been seen that marine sponge secondary metabolites are important as the source of useful anticancer compounds. Among most of the isolated compounds from sponges and their semisynthetic derivatives, there are six compounds

\section{REFERENCES}

Abdel-Lateff, A., Al-Abd, A. M., Alahdal, A. M., Alarif, W. M., Ayyad, S. E., AlLihaibi, S.S. \& Azhar, A. S. (2016). Antiproliferative effects of triterpenoidal derivatives, obtained from the marine sponge Siphonochalina sp., on human hepatic and colorectal cancer cells. Zeitschrift für Naturforschung C, 71(1-2), 29-35. DOI: 10.1515/znc-2015-0160

Bae, S.Y., Kim, G.D., Jeon, J.E., Shin, J. \& Lee, S.K. (2013). Anti-proliferative effect of (19Z)-halichondramide, a novel marine macrolide isolated from the sponge Chondrosia corticata, is associated with G2/M cell cycle arrest and suppression of mTOR signaling in human lung cancer cells. Toxicology In Vitro, 27(2), 694-699.

DOI: 10.1016/j.tiv.2012.11.001

Ben-Califa, N., Bishara, A., Kashman, Y. \& Neumann, D. (2012). Salarin C, a member of the salarin superfamily of marine compounds, is a potent inducer of apoptosis. Investigational New Drugs, 30(1), 98-104. DOI: 10.1007/s10637-010-9521-4

Bérdy, J. (2005). Bioactive Microbial Metabolites. The Journal of Antibiotics, 58(1), 1-26. doi:10.1038/ja.2005.1

Berry, E., Hardt, J.L., Clardy, J., Lurain, J.R. \& Kim, J.J. (2009). Induction of apoptosis in endometrial cancer cells by psammaplysene A involves FOX01. Gynecologic Oncology, 112(2), 331-336. DOI: 10.1016/j.ygyno.2008.10.017

Bhatnagar, I. \& Kim, S.K. (2010). Immense essence of excellence: marine microbial bioactive compounds. Marine Drugs, 8(10), 2673-2701. DOI: $10.3390 / \mathrm{md} 8102673$

Chan, A., Andreae, P.M., Northcote, P.T. \& Miller, J.H. (2011). Peloruside A inhibits microtubule dynamics in a breast cancer cell line MCF7. Investigational New Drugs, 29(4), 615-626. DOI: 10.1007/s10637-010-9398-2

Chen, Y., Zhou, Q., Zhang, L., Zhong, Y., Fan, G., Zhang, Z. \& Kong, D. (2017). Stellettin B induces apoptosis in human chronic myeloid leukemia cells via targeting PI3K and Stat5. Oncotarget, 8(17), 2890628921. DOI: $10.18632 /$ oncotarget.15957

Cheng, X., Li, J., Tanaka, K., Majumder, U., Milinichik, A.Z., Verdi, A.C. \& Albone, E. F. (2018). MORAb-202, an Antibody-Drug Conjugate Utilizing Humanized Anti-human FRalpha Farletuzumab and the Microtubuletargeting Agent Eribulin, has Potent Antitumor Activity. Molecular Cancer Therapeutics, 17(12), 2665-2675. DOI: 10.1158/1535-7163.MCT-17-1215

Cheung, F. W., Li, C., Che, C. T., Liu, B. P., Wang, L., \& Liu, W. K. (2010). Geoditin A induces oxidative stress and apoptosis on human colon HT29 cells. Marine Drugs, 8(1), 80-90. DOI: 10.3390/md8010080

Chinen, T., Nagumo, Y., Watanabe, T., Imaizumi, T., Shibuya, M., Kataoka, T. \& Usui, T. (2010). Irciniastatin A induces JNK activation that is involved in caspase-8-dependent apoptosis via the mitochondrial pathway. Toxicology Letters, 199(3), 341-346. DOI: 10.1016/j.toxlet.2010.09.017

De Stefano, D., Tommonaro, G., Malik, S.A., lodice, C., De Rosa, S., Maiuri, M.C. \& Carnuccio, R. (2012). Cacospongionolide and scalaradial, two marine sesterterpenoids as potent apoptosis-inducing factors in human carcinoma cell lines. PLoS One, 7(4), e33031. DOI: 10.1371/journal.pone.0033031

Dias, D.A., Urban, S. \& Roessner, U. (2012). A historical overview of natural products in drug discovery. Metabolites, 2(2), 303-336. DOI: 10.3390/metabo2020303

Dyshlovoy, S.A., Fedorov, S.N., Shubina, L.K., Kuzmich, A.S., Bokemeyer, C., Keller-von Amsberg, G. \& Honecker, F. (2014). Aaptamines from the marine sponge Aaptos sp. display anticancer activities in human cancer either approved or in clinical phase. Moreover, more than 40 compounds have been tested for anticancer activity in the last 10 years.

The future contribution of marine natural products to cancer treatment seems promising, with a significant improvement of more products from marine sponge secondary metaboites in the clinical and preclinical stages.

cell lines and modulate AP-1-, NF-kappaB-, and p53-dependent transcriptional activity in mouse JB6 $\mathrm{Cl} 41$ cells. BioMed Research International, 2014, 469309.

DOI: $10.1155 / 2014 / 469309$

Dyshlovoy, S.A., Tabakmakher, K.M., Hauschild, J., Shchekaleva, R.K., Otte, K., Guzii, A.G. \& von Amsberg, G. (2016a). Guanidine Alkaloids from the Marine Sponge Monanchora pulchra Show Cytotoxic Properties and Prevent EGF-Induced Neoplastic Transformation in Vitro. Marine Drugs, 14(7).

DOI: $10.3390 / \mathrm{md} 14070133$

Dyshlovoy, S.A., Otte, K., Alsdorf, W.H., Hauschild, J., Lange, T., Venz, S. \& von Amsberg, G. (2016b). Marine compound rhizochalinin shows high in vitro and in vivo efficacy in castration resistant prostate cancer. Oncotarget, 7(43), 69703-69717. DOI: $10.18632 /$ oncotarget.11941

Ebada, S.S., Wray, V., De Voogd, N.J., Deng, Z., Lin, W. \& Proksch, P. (2009). Two New Jaspamide Derivatives from the Marine Sponge Jaspis splendens. Marine Drugs, 7(3), 435-444. DOI: 10.3390/md7030435

Edelson, J.R., \& Brautigan, D.L. (2011). The Discodermia calyx toxin calyculin a enhances cyclin D1 phosphorylation and degradation, and arrests cell cycle progression in human breast cancer cells. Toxins (Basel), 3(1), 105-119. DOI: 10.3390/toxins3010105

Fedorov, S.N., Makarieva, T.N., Guzii, A.G., Shubina, L.K., Kwak, J.Y. \& Stonik, V.A. (2009). Marine two-headed sphingolipid-like compound rhizochalin inhibits EGF-induced transformation of JB6 P+ Cl41 cells. Lipids, 44(9), 777-785. DOI: $10.1007 /$ s11745-009-3322-6

Florean, C., Schnekenburger, M., Lee, J.Y., Kim, K.R., Mazumder, A., Song, S. \& Diederich, M. (2016). Discovery and characterization of Isofistularin-3, a marine brominated alkaloid, as a new DNA demethylating agent inducing cell cycle arrest and sensitization to TRAIL in cancer cells. Oncotarget, 7(17), 24027-24049. DOI: $10.18632 /$ oncotarget.8210

Gesto, D.S., Cerqueira, M.F.S.A., Fernandes, P.A. \& Ramos, M. J. (2012). Gemcitabine: A Critical Nucleoside for Cancer Therapy. Current Medicinal Chemistry, 19(7), 1076-1087. DOI: $10.2174 / 092986712799320682$

Guzman, E.A., Johnson, J.D., Carrier, M.K., Meyer, C.I., Pitts, T. P., Gunasekera, S.P. \& Wright, A. E. (2009). Selective cytotoxic activity of the marine-derived batzelline compounds against pancreatic cancer cell lines. Anticancer Drugs, 20(2), 149-155 DOI: 10.1097/CAD.0b013e32831fa39e

Guzman, E., Maher, M., Temkin, A., Pitts, T. \& Wright, A. (2013). Spongiatriol inhibits nuclear factor kappa B activation and induces apoptosis in pancreatic cancer cells. Marine Drugs, 11(4), 1140-1151. DOI: $10.3390 / \mathrm{md} 11041140$

Guzman, E.A., Maers, K., Roberts, J., Kemami-Wangun, H.V., Harmody, D. \& Wright, A.E. (2015). The marine natural product microsclerodermin A is a novel inhibitor of the nuclear factor kappa $B$ and induces apoptosis in pancreatic cancer cells. Investigational New Drugs, 33(1), 86-94. DOI: 10.1007/s10637-014-0185-3

Guzman, E.A., Xu, Q., Pitts, T.P., Mitsuhashi, K.O., Baker, C., Linley, P.A. \& Wright, A.E. (2016). Leiodermatolide, a novel marine natural product, has potent cytotoxic and antimitotic activity against cancer cells, appears 
to affect microtubule dynamics, and exhibits antitumor activity International Journal of Cancer, 139(9), 2116-2126. DOI: 10.1002/ijc.30253

Halim, H., Chunhacha, P., Suwanborirux, K. \& Chanvorachote, P. (2011). Anticancer and antimetastatic activities of Renieramycin $M$, a marine tetrahydroisoquinoline alkaloid, in human non-small cell lung cancer cells. Anticancer Research, 31(1), 193-201.

Huang, H.H., Kuo, S.M., Wu, Y.J. \& Su, J.H. (2016). Improvement and enhancement of antibladder carcinoma cell effects of heteronemin by the nanosized hyaluronan aggregation. International Journal of Nanomedicine, 11, 1237-1251. DOI: 10.2147/ijn.S99911

Jin, J.O., Shastina, V., Park, J.I., Han, J.Y., Makarieva, T., Fedorov, S. \& Kwak, J.Y. (2009). Differential induction of apoptosis of leukemic cells by rhizochalin, two headed sphingolipids from sponge and its derivatives. Biological and Pharmaceutical Bulletin, 32(6), 955-962.

Khanal, P., Kang, B.S., Yun, H.J., Cho, H.G., Makarieva, T.N. \& Choi, H.S (2011). Aglycon of rhizochalin from the Rhizochalina incrustata induces apoptosis via activation of AMP-activated protein kinase in HT-29 colon cancer cells. Biological and Pharmaceutical Bulletin, 34(10), 1553-1558.

Kong, D., Aoki, S., Sowa, Y., Sakai, T. \& Kobayashi, M. (2008). Smenospongine, a sesquiterpene aminoquinone from a marine sponge, induces G1 arrest or apoptosis in different leukemia cells. Marine Drugs, 6(3), 480-488. DOI: 10.3390/md20080023

Konishi, H., Kikuchi, S., Ochiai, T., Ikoma, H., Kubota, T., Ichikawa, D. \& Otsuji, E. (2009). Latrunculin a has a strong anticancer effect in a peritoneal dissemination model of human gastric cancer in mice. Anticancer Research, 29(6), 2091-2097.

Krauss, A.C., Gao, X., Li, L., Manning, M.L., Patel, P., Fu, W. \& Pazdur, R. (2018). FDA Approval Summary: (Daunorubicin and Cytarabine) Liposome for Injection for the Treatment of Adults with High-Risk Acute Myeloid Leukemia. Clinical Cancer Research., 25(9), 2685-2690 DOI: 10.1158/1078-0432.Ccr-18-2990

Leal, M.C., Madeira, C., Brandao, C. A., Puga, J. \& Calado, R. (2012) Bioprospecting of marine invertebrates for new natural products - a chemical and zoogeographical perspective. Molecules, 17(8), 9842 9854. DOI:10.3390/molecules 17089842

Lee, H.Y., Chung, K.J., Hwang, I. H., Gwak, J., Park, S., Ju, B.G. \& Oh, S. (2015). Activation of p53 with ilimaquinone and ethylsmenoquinone, marine sponge metabolites, induces apoptosis and autophagy in colon cancer cells. Marine Drugs, 13(1), 543-557. DOI: $10.3390 / \mathrm{md} 13010543$

Liu, W.K., Ho, J.C. \& Che, C.T. (2005). Apoptotic activity of isomalabaricane triterpenes on human promyelocytic leukemia HL60 cells. Cancer Letters, 230(1), 102-110. DOI: $10.1016 /$ j.canlet.2004.12.034

Livett, B.G., Gayler, K.R. \& Khalil, Z. (2004). Drugs from the Sea: Conopeptides as Potential Therapeutics. Current Medicinal Chemistry, 11(13), 1715-1723. DOI: 10.2174/0929867043364928

Mailhol, D., Willwacher, J., Kausch-Busies, N., Rubitski, E.E., Sobol, Z., Schuler, M., Lam, M.-H., Musto, S., Loganzo,F., Maderna, A. \& Furstner, A. (2014). Synthesis, molecular editing, and biological assessment of the potent cytotoxin leiodermatolide. Journal of the American Chemical Society, 136(44), 15719-15729. DOI: $10.1021 / \mathrm{ja} 508846 \mathrm{~g}$

Malve, H. (2016). Exploring the ocean for new drug developments: Marine pharmacology. Journal of Pharmacy And Bioallied Sciences, 8(2), 83-91. DOI: 10.4103/0975-7406.171700

Mann, J. (1994). Natural products: their chemistry and biological significance. Harlow, Essex, England; New York: Longman Scientific \& Technical Wiley.

Martins, A., Vieira, H., Gaspar, H. \& Santos, S. (2014). Marketed marine natural products in the pharmaceutical and cosmeceutical industries: tips for success. Marine Drugs, 12(2), 1066-1101. DOI: $10.3390 / \mathrm{md} 12021066$

Menis, J. \& Twelves, C. (2011). Eribulin (Halaven): a new, effective treatment for women with heavily pretreated metastatic breast cancer. Breast Cancer (Dove Med Press), 3, 101-111. DOI: $10.2147 /$ BCTT.S21741
Mishra, B.B., \& Tiwari, V.K. (2011). Natural products: an evolving role in future drug discovery. European Journal of Medicinal Chemistry, 46(10), 4769-4807. DOI: 10.1016/j.ejmech.2011.07.057

Molinski, T.F., Dalisay, D.S., Lievens, S.L. \& Saludes, J.P. (2009). Drug development from marine natural products. Nature Reviews Drug Discovery, 8(1), 69-85. DOI: 10.1038/nrd2487

Nguyen, H.T., Minh, C.V., Ha, T.T., Kiem, P., V., Huong, T.H., Dat, N.T., Nhiem, N.X., Tai, B.H., Hyun, J.-H.,Kang, H.-K. \& Kim, Y.H. (2009). C29 sterols with a cyclopropane ring at $\mathrm{C}-25$ and 26 from the Vietnamese marine sponge lanthella sp. and their anticancer properties. Bioorganic \& Medicinal Chemistry Letters, 19(16), 4584-4588. DOI: 10.1016/j.bmcl.2009.06.097

Pantazopoulou, A., Galmarini, C.M. \& Peñalva, M.A. (2018). Molecular basis of resistance to the microtubule-depolymerizing antitumor compound plocabulin. Scientific Reports, 8(1), 8616 . DOI: 10.1038/s41598-018-26736-3

Paterson, I., Dalby, S.M., Roberts, J.C., Naylor, G.J., Guzman, E.A., Isbrucker, R., Pitts, T.P., Linley, P., Divlianska, D., Reed, J.K. \& Wright, A.E. (2011). Leiodermatolide, a potent antimitotic macrolide from the marine sponge Leiodermatium sp. Angewandte Chemie International Edition, 50(14), 3219-3223. DOI: 10.1002/anie.201007719

Pettit, G.R., Fujii, Y., Hasler, J.A., \& Schmidt, J.M. (1982). Isolation and Characterization of Palystatins A-D. Journal of Natural Products, 45(3), 272-276. DOI:10.1021/np50021a007

Pihlanto-Leppälä, A. (2000). Bioactive peptides derived from bovine whey proteins: opioid and ace-inhibitory peptides. Trends in Food Science \& Technology, 11(9), 347-356. DOI: 10.1016/S0924-2244(01)00003-6

Radwan, M., Hanora, A., Khalifa, S. \& Abou-El-Ela, S.H. (2012) Manzamines: a potential for novel cures. Cell Cycle, 11(9), 1765-1772. DOI: $10.4161 /$ cc.20135

Rasmussen, R.S., \& Morrissey, M.T. (2007). Marine Biotechnology for Production of Food Ingredients. In Advances in Food and Nutrition Research (Vol. 52, pp. 237-292): Academic Press.

Rath, B., Hochmair, M., Plangger, A. \& Hamilton, G. (2018). Anticancer Activity of Fascaplysin against Lung Cancer Cell and Small Cell Lung Cancer Circulating Tumor Cell Lines. Marine Drugs, 16(10), 383 DOI: $10.3390 / \mathrm{md} 16100383$

Roel, M., Rubiolo, J.A., Guerra-Varela, J., Silva, S.B., Thomas, O.P. Cabezas-Sainz, P.,Sánchez, L., López, R. \& Botana, L.M. (2016). Marine guanidine alkaloids crambescidins inhibit tumor growth and activate intrinsic apoptotic signaling inducing tumor regression in a colorectal carcinoma zebrafish xenograft model. Oncotarget, 7(50), 83071-83087.

DOI: 10.18632/oncotarget.13068

Rothmeier, A. S., Schneiders, U.M., Wiedmann, R.M., Ischenko, I., Bruns, C. J., Rudy, A., Zahler, S. \& Vollmar, A.M. (2010). The marine compound spongistatin 1 targets Vollmar, A.M. (2010). The marine compound spongistatin 1 targets pancreatic tumor progression and metastasis. International Journal of Cancer, 127(5), 1096-1105. DOI: $10.1002 /$ ijc. 25241

Salma, Y., Lafont, E., Therville, N., Carpentier, S., Bonnafe, M.J., Levade, T. Génisson, Y. \& Andrieu-Abadie, N. (2009). The natural marine anhydrophytosphingosine, Jaspine $B$, induces apoptosis in melanoma cells by interfering with ceramide metabolism. Biochemical Pharmacology, 78(5), 477-485.

DOI: 10.1016/j.bcp.2009.05.002

Schneiders, U.M., Schyschka, L., Rudy, A. \& Vollmar, A.M. (2009). BH3-only proteins $\mathrm{Mcl}-1$ and Bim as well as endonuclease $\mathrm{G}$ are targeted in spongistatin 1-induced apoptosis in breast cancer cells. Molecular Cancer Therapeutics, 8(10), 2914-2925 DOI: 10.1158/1535-7163.Mct-08-1179

Schumacher, M., Cerella, C., Eifes, S., Chateauvieux, S., Morceau, F., Jaspars, M., Dicato, M. \& Diederich, M. (2010). Heteronemin, a spongean sesterterpene, inhibits TNF alpha-induced NF-kappa B activation through proteasome inhibition and induces apoptotic cell death. Biochemical Pharmacology, 79(4), 610-622. DOI: 10.1016/j.bcp.2009.09.027 
Schumacher, M., Kelkel, M., Dicato, M. \& Diederich, M. (2011). Gold from the sea: marine compounds as inhibitors of the hallmarks of cancer. Biotechnology Advances, 29(5), 531-547. DOI: 10.1016/j.biotechadv.2011.02.002

Sobahi, T.R.A., Ayyad, S.N., Abdel-Lateff, A., Algandaby, M.M., Alorfi, H.S. \& Abdel-Naim, A.B. (2017). Cytotoxic Metabolites from Callyspongia siphonella Display Antiproliferative Activity by Inducing Apoptosis in HCT-116 Cells. Pharmacognosy Magazine, 13(Suppl 1), S37-s40. DOI: $10.4103 / 0973-1296.203970$

Su, J.-H., Chang, W.-B., Chen, H.-M., El-Shazly, M., Du, Y.-C., Kung, T.-H., Chen, Y. C., Sung, P.-J., Ho, Y.-S., Kuo, F.-W. \& Lu, M.-C. (2012). 10 Acetylirciformonin B, A Sponge Furanoterpenoid, Induces DNA Damage and Apoptosis in Leukemia Cells. Molecules, 17(10), 11839-11848. DOI:10.3390/molecules171011839

Sudek, S., Lopanik, N.B., Waggoner, L.E., Hildebrand, M., Anderson, C., Liu H. \& Haygood, M.G. (2007). Identification of the Putative Bryostatin Polyketide Synthase Gene Cluster from "Candidatus Endobugula sertula", the Uncultivated Microbial Symbiont of the Marine Bryozoan Bugula neritina. Journal of Natural Products, 70(1), 67-74. DOI:10.1021/np060361d

Tang, S.A., Zhou, Q., Guo, W.Z., Qiu, Y., Wang, R., Jin, M. \& Kong, D. (2014). In vitro antitumor activity of stellettin $B$, a triterpene from marine sponge Jaspis stellifera, on human glioblastoma cancer SF295 cells. Marine Drugs, 12(7), 4200-4213. DOI: 10.3390/md12074200

Teta, R., Irollo, E., Della Sala, G., Pirozzi, G., Mangoni, A. \& Costantino, V. (2013). Smenamides A and B, chlorinated peptide/polyketide hybrids containing a dolapyrrolidinone unit from the Caribbean sponge Smenospongia aurea. Evaluation of their role as leads in antitumor drug research. Marine Drugs, 11(11), 4451-4463. DOI: $10.3390 / \mathrm{md} 11114451$

Thomas, T.R., Kavlekar, D.P. \& LokaBharathi, P.A. (2010). Marine drugs from sponge-microbe association--a review. Marine Drugs, 8(4), 1417 1468. DOI: $10.3390 / \mathrm{md} 8041417$

Torre, L.A., Bray, F., Siegel, R.L., Ferlay, J., Lortet-Tieulent, J. \& Jemal, A (2015). Global cancer statistics, 2012. A Cancer Journal for Clinicians, 65(2), 87-108. DOI:10.3322/caac. 21262
Umeyama, A., Matsuoka, N., Mine, R., Nakata, A., Arimoto, E., Matsui, M. \& Hashimoto, T. (2010). Polyacetylene diols with antiproliferative and driving Th1 polarization effects from the marine sponge Callyspongia sp. Journal of Natural Medicines, 64(1), 93-97.

DOI: 10.1007/s11418-009-0363-3

Wang, W., Rayburn, E.R., Velu, S.E., Nadkarni, D.H., Murugesan, S. \& Zhang, R. (2009). In vitro and in vivo anticancer activity of novel synthetic makaluvamine analogues. Clinical Cancer Research, 15(10), 3511-3518. DOI: 10.1158/1078-0432.Ccr-08-2689

Wang, L., Li, D., Xu, S., Cai, H., Yao, H., Zhang, Y., Jiang, J. \& Xu, J. (2012). The conversion of oridonin to spirolactone-type or enmein-type diterpenoid: synthesis and biological evaluation of ent-6,7-seco-oridonin derivatives as novel potential anticancer agents. European Journal of Medicinal Chemistry, 52, 242-250. DOI: 10.1016/j.ejmech.2012.03.024

Wang, R., Zhang, Q., Peng, X., Zhou, C., Zhong, Y., Chen, X., Qiu, Y., Jin, M., Gong, M. \& Kong, D. (2016). Stellettin B Induces G1 Arrest, Apoptosis and Autophagy in Human Non-small Cell Lung Cancer A549 Cells via Blocking PI3K/Akt/mTOR Pathway. Scientific Reports, 6, 27071. DOI: 10.1038/srep27071

Whitson, E.L., Pluchino, K.M., Hall, M.D., McMahon, J.B. \& McKee, T.C. (2011). New candidaspongiolides, tedanolide analogues that selectively inhibit melanoma cell growth. Organic Letters, 13(13), 3518-3521. DOI: 10.1021/ol201329p

Wu, J.C., Wang, C.T., Hung, H.C., Wu, W.J., Wu, D.C., Chang, M.C. \& Tai M. H. (2016). Heteronemin Is a Novel c-Met/STAT3 Inhibitor Against Advanced Prostate Cancer Cells. The Prostate, 76(16), 1469-1483. DOI: $10.1002 /$ pros.23230

Yoo, H., Lee, Y.S., Lee, S., Kim, S. \& Kim, T.Y (2012). Pachastrissamine from Pachastrissa $\mathrm{sp}$. inhibits melanoma cell growth by dual inhibition of Cdk2 and ERK-mediated FOXO3 downregulation. Phytotherapy Research, 26(12), 1927-1933. DOI: 10.1002/ptr.4673

Zhang, Y.W., Ghosh, A.K. \& Pommier, Y. (2012). Lasonolide A, a potent and reversible inducer of chromosome condensation. Cell Cycle, 11(23), 4424-4435. DOI: $10.4161 /$ cc. 22768 